\title{
UAV-BASED OBLIQUE PHOTOGRAMMETRY FOR 3D RECONSTRUCTION OF TRANSMISSION LINE: PRACTICES AND APPLICATIONS
}

\author{
San Jiang ${ }^{1}$, Wanshou Jiang ${ }^{23 *}$ \\ ${ }^{1}$ School of Computer Science, China University of Geosciences, Wuhan 430074, China - jiangsan@cug.edu.cn \\ ${ }^{2}$ State Key Laboratory of Information Engineering in Surveying, Mapping and Remote Sensing, Wuhan University, Wuhan 430072, China \\ ${ }^{3}$ Collaborative Innovation Center of Geospatial Technology, Wuhan University, Wuhan, 430072, China - jws@ whu.edu.cn
}

Commission II, WG II/4

KEY WORDS: Unmanned Aerial Vehicle, Oblique Photogrammetry, 3D Reconstruction, Dual-camera, Dual-strip, Transmission Line

\begin{abstract}
:
Regular inspection of transmission line is an essential work to ensure reliable supply and distribution of electric power. Considering that the conventional time consuming and economic inefficient methods can not satisfy new coming demands, an inspection strategy is proposed in this study, which aims to achieve off-site visual inspection instead of in-site inspection. The most challenging task is 3D model reconstruction of transmission line. Therefore, based on oblique imaging technique and the flexibility of UAV platforms, an UAV-Based oblique photogrammetric system is designed and manufactured for data acquisition of transmission line. The system integrates a dual-camera imaging system, an onboard GNSS (Global Navigation Satellite System) receiver and a ground fixed GNSS station. Beside, using open-source and commercial software packages, a data processing pipeline is also proposed for 3D model reconstruction. The experimental results demonstrate that the UAV-based oblique imaging system could obtain images of power pylons in four different directions and provides enough information for the successful model reconstruction. Without GCPs, the dual-strip flight mode is recommended for flight configuration to achieve high geo-referencing accuracy. Compared with the traditional methods, the proposed solution can achieve off-site inspection of transmission line.
\end{abstract}

\section{INTRODUCTION}

Regular inspection of transmission line is an essential work (Ahmad et al., 2013), and it is dominated by inspecting vegetation encroachment. The most common approaches utilized by most electricity companies are foot patrol and helicopter-assisted inspection. However, these two methods have obvious drawbacks. On the one hand, foot patrol is labour intensive and time consuming, and high economic costs are usually required for helicopterassisted inspection, such as integration with ALS (Airborne Laser Scanner) systems. On the other hand, a vast majority of transmission lines locates in mountainous regions, which could cause too much false inspection because of occlusions of trees and lack of experiences, etc. Thus, with the expansion of power grid, these traditional solutions can more and more not satisfy new coming demands (Katrasnik et al., 2009).

Instead of in-site inspection, off-site inspection could be an alternative and reliable approach, which can be achieved by $3 \mathrm{D}$ models of transmission line. 3D models of power pylons and lines are capable for transmission line management in many aspects, including geometry calculation of power pylons for electrical parameter prediction, distance measurement between power lines and vegetation for encroachment inspection, etc. Compared with the traditional manual operation methods, this strategy could provide accurate measurement ability to obtain essential information for transmission line inspection under 3D measurable environment. In the literature, some new techniques have emerged for $3 \mathrm{D}$ reconstruction of transmission line.

In recent years, oblique images are becoming increasingly more important in photogrammetry field, and a wide range of appli-

\footnotetext{
${ }^{*}$ Corresponding author
}

cations has reported (Remondino and Gerke, 2015). Comparing with the traditional photogrammetry, oblique imaging technique could capture both footprints and facades of target objects, which can dramatically facilitate the relating applications, including dense matching (Gerke, 2009), damaged building detection (Gerke and Kerle, 2015, Vetrivel et al., 2015), land coverage analysis (Rau et al., 2014) and 3D building model reconstruction (Yalcin and Selcuk, 2015, Zhu et al., 2015), and so on. With the improvement of aerial multi-camera systems, the development of oblique photogrammetry has been promoted and some more applications have been documented (Rupnik et al., 2015).

While a diversity of applications of oblique photogrammetry have been reported in both literature and civil fields, the platforms used in the above data acquisition are aerial planes, which is not flexible and convenient for data campaigns of small regions or corridors, such as transmission line (Jiang et al., 2017). As a complementary for the conventional aerial photogrammetry, unmanned aerial vehicle (UAV) has been emerged and utilized in many applications (Boccardo et al., 2015, Gonçalves and Henriques, 2015, Lin et al., 2015) because of easy operation, low costs and the ability for flexible data acquisition (Nex and Remondino, 2014). Hence, the integration of oblique imaging technique and UAV platform could lead to an useful oblique photogrammetric system for data acquisition in some special applications, for example 3D reconstruction of transmission line.

In this paper, we would first describe a design of an UAV based oblique photogrammetric system. Second, a dual-strip flight mode is used for data acquisition of transmission. Then, 3D models are reconstructed under manual digitization. Finally, some applications are presented by using reconstructed models. The workflow is presented in Figure 1. 


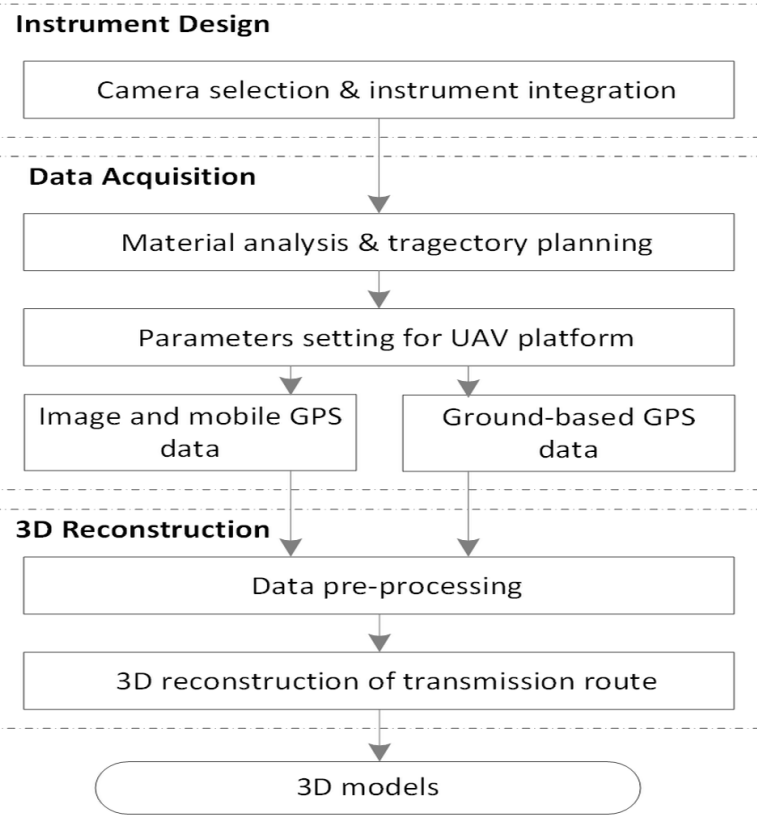

Figure 1. The workflow for 3D model reconstruction of transmission line.

\section{PHOTOGRAMMETRIC SYSTEM AND 3D MODEL RECONSTRUCTION PIPELINE}

\subsection{Oblique Photogrammetric System}

In order to facilitate 3D model reconstruction of power pylons, images should be captured from four directions with respective to power pylons, which contain information in the front, back, left and right directions. Besides, because of the elongated structure of the transmission line corridor, it is not convenient and time consuming to conduct multi-flights to record enough images.

In this study, an oblique photogrammetric system, integrating a dual-camera imaging system, an onboard dual-frequency GNSS (Global Navigation Satellite System) receiver and a ground master GNSS station in fixed position, is designed and integrated with a multi-rotor UAV, which is illustrated in Figure 2. The onboard GNSS receiver and the ground fixed GNSS station are used for differential GNSS (DGNSS) calculation. The onboard GNSS receiver is mounted at the top of UAV platform and the dual-camera system is installed at the bottom of the platform. Considering image resolution and payload limitation of the UAV platform, two Sony RX1R digital cameras with 24Mpixel sensor, $35 \mathrm{~mm}$ focal length are chosen to design image record instrument.

To adapt to different flight height, the mounting angles of the dual-camera system, including pitch and roll angles, can be adjusted to make sure that power pylons are imaged in the central location of captured photos. For one data campaign, one came termed front camera is used to capture images in the alongtrack direction. The other camera termed back camera is adopted for image acquisition in the across-track direction. Therefore, enough images can be obtained within one flight campaign.

\subsection{Data Acquisition and Pre-processing}

Three major steps should be conducted for data acquisition:

(1) Some materials about test sites, including height of power pylon, topographical change of terrain, are collected and analysed.

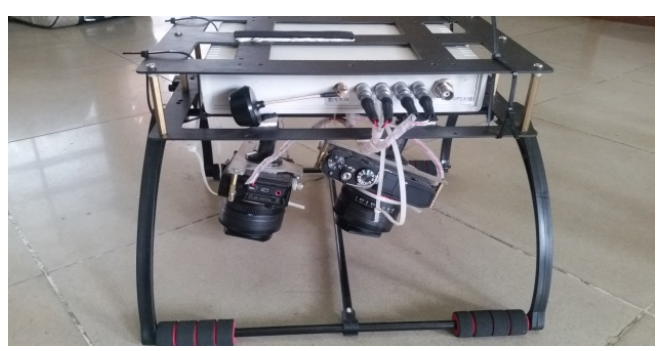

(a) Dual-camera imaging system

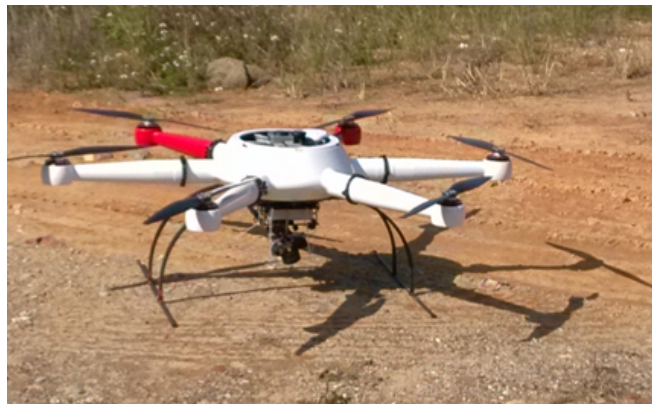

(b) UAV platform

Figure 2. Oblique photogrammetric system.

(2) Flight configuration should be designed. According to the height of power pylons, flight height can be determined by adding an offset value to the height of power pylons where the offset set value is in the range between 35 and $60 \mathrm{~m}$. meanwhile, a safe flight distance is used to determine the horizontal distance, which is perpendicular to transmission line. Then, based on oblique imaging geometry, camera mounting angles can be calculated with the restriction that power pylons should be recorded near the central location of photos.

(3) The last step is to conduct the outdoor data campaign with the configured oblique photogrammetry system. Because most UAV platforms are capable of automatic cruising mode, a flight trajectory is generated prior to the outdoor campaign, which could decrease the manual intervention. Because transmission lines are usually located in mountainous areas, the topological change should be seriously considered to determine flight height. In this study, the generated flight height is adapted to terrain height, where the relative height between the UAV platform and power pylons is almost consistent.

In order to enhance image connection in the across-track direction and obtain enough images of power pylons, a dual-strip flight mode is utilized in this study, which is illustrated in Figure3. Cooperating with the dual-camera system, images of power pylons can be captured from four different directions. Advantages of the dual-strip flight mode would be verified in the experimental section.

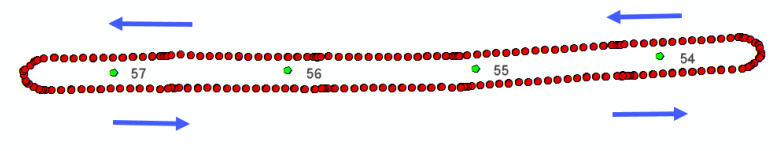

Figure 3. Dual-strip flight mode for data acquisition. Red circles are image positions. Green circles are locations of power pylons. Blue arrows stand for UAV flying directions. 
After data acquisition, images and GNSS data are obtained. To improve geo-referencing accuracy, the original GNSS data from both the onboard and the ground fixed receivers are processed based on differential GNSS (DGNSS). In this study, differential GNSS operation is conducted by using the GrafNav software.

During data acquisition, the central control unit has recorded exposure time of each image. To decrease geo-referencing error caused by the delay between GPS signal receiving and photo capture, each image's position is refined by linear interpolation using time information as presented by Equation (1), where $T_{i m g}$ indicates image capture time; $T_{g p s 1}$ and $T_{g p s 2}$ stand for two successive GNSS times that are closest to $T_{i m g} ; P_{g p s 1}, P_{g p s 2}$ and $P_{i m g}$ are positions corresponding to the three times.

$$
P_{i m g}=P_{g p s 1}+\frac{T_{i m g}-T_{g p s 1}}{T_{g p s 2}-T_{g p s 1}} P_{g p s 2}
$$

\subsection{D Model Reconstruction Pipeline}

2.3.1 Image orientation For the purpose of transmission line inspection, 3D models contain terrain model of corridor and models relating to electric facilities, which mainly consist of power pylons and lines. For all these above mentioned models, image orientation is the first step within the pipeline of 3D model reconstruction.

In this study, the open-source software package, MicMac (Pierrot Deseilligny and Clery, 2012), is adopted for image orientation. Firstly, SIFT features are extracted for each image. Secondly, both image positions generated from DGNSS and camera mounting angles are used to select the initial match pairs, which are overlapped. Image matching is guided by the initial match pairs where only image pairs in the match pair set can be chose for SIFT feature matching. To decrease time consuming in these two steps, the open-source library SiftGPU (Wu, 2007) is used. After outliers remove based on geometric estimation of a fundamental matrix, feature matches are finally used in the tool Apero for image orientation where image positions are used for georeferencing.

2.3.2 Terrain reconstruction Some commercial software packages, including Leica LPS, Pixel4UAV and PhotoScan, provide complete solution for terrain reconstruction, which follow traditional photogrammetric workflow that starts from camera calibration, automatic or manual tie-point extraction and DSM generation after image orientation.

Recently, Smart3DCapture developed by Acute3D Corporation has captured amount of attention from fields of digital photogrammetry and computer vision, which has been successfully utilized in the fields of digital city, heritage conservation and archeology documentation. Smart3DCapture is characterized full automation, high reality and efficiency. Thus, Smart3DCapture is selected in this study for terrain reconstruction. Additionally, some new tools are created to transform image orientation results, consisting of camera intrinsic calibration, tie-points and orientation, to exchangeable $\mathrm{xml}$ file which can be imported into Smart3DCapture. Then, the conventional procedures are conducted to generate 3D models of transmission corridor, which includes dense matching, triangular meshing and texture mapping as presented in Figure 4. In this study, the generated models are in format of osgb, which could be loaded into many commercial and open-source 3D GIS platform, and LOD (Level of Detail) has been created to speed up scene navigation.

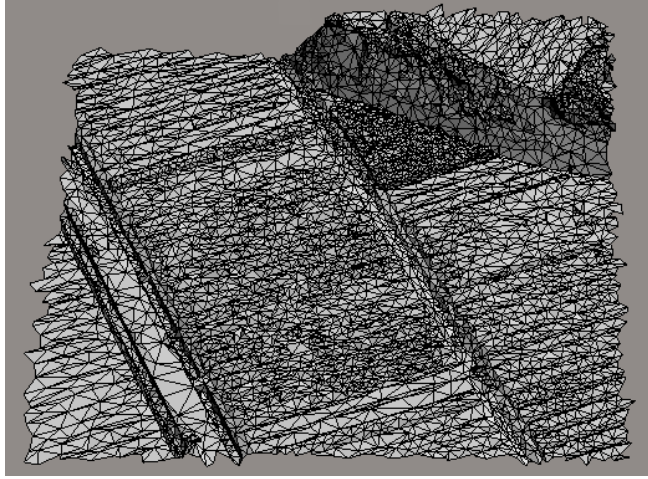

(a) Triangular meshing

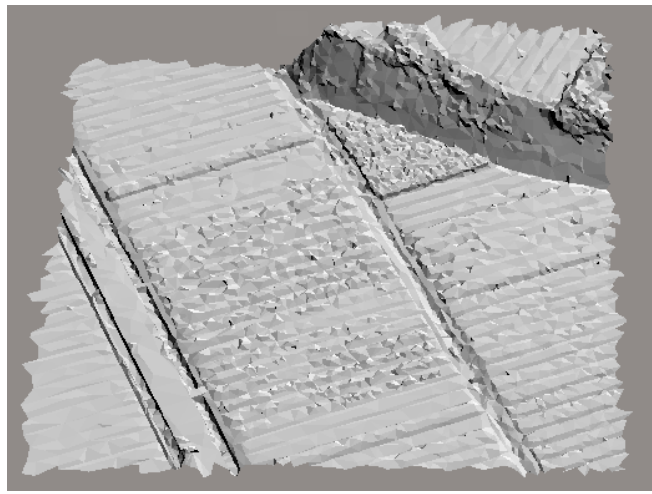

(b) Shaded relief

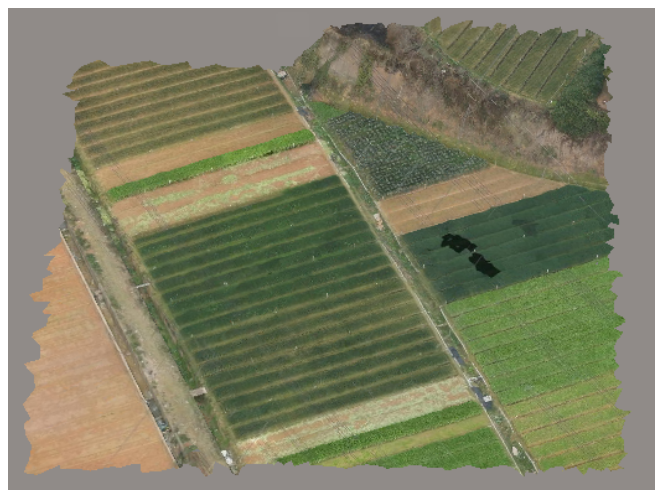

(c) Texture mapping

Figure 4. Reconstruction procedure.

2.3.3 Power pylon and line reconstruction A new semiautomatic pipeline has been promoted in this study for reconstruction of power pylon and line, and an application is designed and developed as shown in Figure 5. First, images captured from different viewpoints, especially in opposite direction, are automatically loaded based on geo-location. Then, operators select some best photos and digitize the base and body of a power pylon with some intelligent tools, such as horizontal and vertical constrains, symmetric operations, etc. Finally, some affiliated components are added to the tower body, which mainly consist of insulators, hanging points of power lines. For reconstruction of power lines, two or three sampled points have been recorded, which are usually closed to hanging points of power lines. Under above operations, a power pylon would be modelled with power line fitted by catenary curves . This application provides some practical tools for model reconstruction, which are simple to operate and labour relief. 


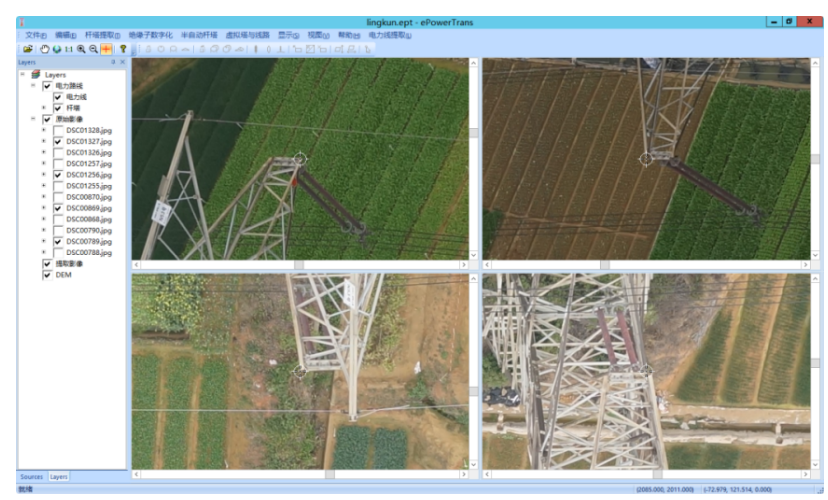

Figure 5. Main UI of the applications for 3D reconstruction of power pylons and lines.

\section{EXPERIMETNS AND RESULTS}

\subsection{Dataset}

The tests are performed with a dataset captured in Shenzhen, China. Most part of the selected test site is covered by farmlands and four power pylons and several roads exist in this region. The height of power pylon is about $65 \mathrm{~m}$ and the flight height is set as $120 \mathrm{~m}$ with respective to ground. In order to record images of power pylons, the pitch and roll angles of the front camera are set as $25^{\circ}$ and $-15^{\circ}$, respectively. Similarly, the angles for the back camera are $0^{\circ}$ and $-25^{\circ}$. Under the flight configuration, $542 \mathrm{im}$ ages with a resolution $3.67 \mathrm{~cm}$ are captured. Figure 6 shows the images of one power pylon in four different directions. We can see that enough information of the power pylon is recorded from the dual-camera imaging system and the dual-strip flight mode, in which steel structures can be clearly observed. Additionally, to evaluate geo-referencing accuracy of the proposed solution, 43 ground control points (GCP) are selected and measured, which are mainly located in the roads and some water pools.

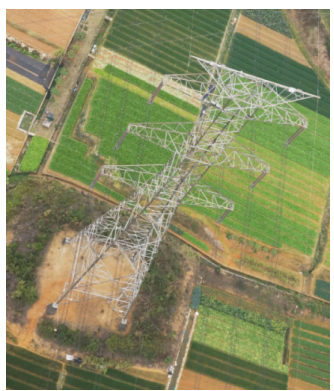

(a) Front

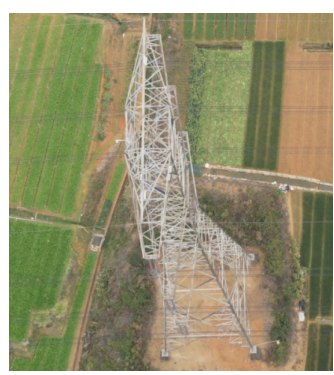

(c) Left

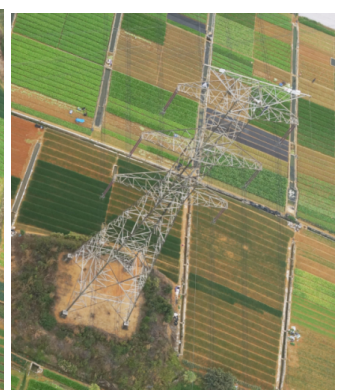

(b) Back

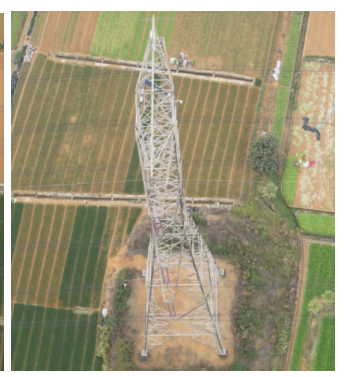

(d) Right
Figure 6. Images of one power pylon in four directions.

\subsection{Image Orientation}

Image orientation for this test site is performed with full automation and the orientation result is presented in Figure 7. With consideration of image positions and camera mounting angles for match pair selection, image orientation has been achieved with root mean square re-projection error (RMSE) near 0.77 pixels, and all images are connected.

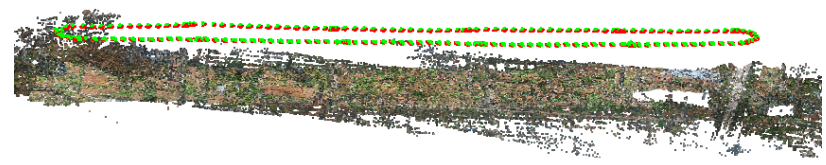

Figure 7. Image orientation of the test site with the assistant of image locations and camera mounting angles.

Single-strip and dual-strip are two different UAV flight modes for data acquisition of transmission line. Single-strip consists of only one flight from one power pylon to the other. Usually, aerial vehicles fly overhead transmission line (Sun et al., 2006). On the contrary, dual-strip is a closed loop flight mode where aerial vehicles usually fly besides transmission line.

A comparing test between these two flight modes is conducted. Images captured in the direction from power pylon labelled by 54 to 57 are selected to simulate single-strip data acquisition mode as shown in Figure 3. A separate image orientation has been done with image positions. 3D point cloud of tie-points from singlestrip and dual-strip experiments are overlapped as shown in Figure 8 . The red coloured point cloud is generated in single-strip experiment and the green coloured point cloud is from dual-strip experiment. Obviously, a roll transformation is existed between these two point clouds.

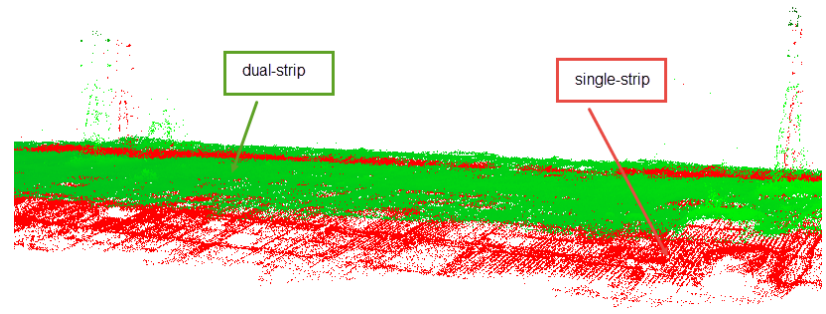

Figure 8. Overlap display of 3D point cloud of tie-points from single-strip and dual-strip.

All measured ground truth points are used as check points to evaluate image orientation accuracy for single-strip and dual-strip flight modes. Evaluation results are listed in Table 1. Under the single-strip flight mode, the mean orientation residuals in $\mathrm{X}, \mathrm{Y}$ and $\mathrm{Z}$ directions are $21.55 \mathrm{~m}, 15.30 \mathrm{~m}$ and $10.22 \mathrm{~m}$, respectively. Noticeable improvement for orientation accuracy has been achieved in the dual-strip mode where the mean residuals decrease to $0.35 \mathrm{~m}, 0.20 \mathrm{~m}$ and $0.82 \mathrm{~m}$, respectively. The main reason is that image pairs in across-track direction constrain roll angle and ensure that it is not arbitrary during image pose estimation.

The mean residuals of image orientation without GCPs could be better than $0.4 \mathrm{~m}$ in horizontal direction and $0.9 \mathrm{~m}$ in vertical direction under dual-strip mode. This is a promising result for transmission line inspection due to the fact that almost all transmission lines are located in mountainous regions, where ground 
control points rarely could be measured. Therefore, the dual-strip flight mode is recommended for purpose of transmission line inspection without GCPs.

Table 1. Accuracy comparison of bundle adjustment (in meters).

\begin{tabular}{lllllll}
\hline \multirow{2}{*}{ Mode } & \multicolumn{2}{l}{ Average } & \multicolumn{4}{l}{ Stdev } \\
\cline { 2 - 7 } & $|X|$ & $|Y|$ & $|Z|$ & $X$ & $Y$ & $Z$ \\
\hline Single & 21.55 & 15.30 & 10.22 & 5.29 & 3.05 & 10.01 \\
Dual & 0.35 & 0.20 & 0.82 & 0.08 & 0.13 & 0.24 \\
\hline
\end{tabular}

\subsection{D Model Reconstruction}

The reconstructed models of corridor terrain, power pylons and lines can be used for physical dimension calculation as well as visualization. Based on osgEarth software development toolkit, a free and open-source 3D GIS platform, a new software package has been developed for visualization and spatial analysis of transmission line as shown in Figure 9, which provides abilities for measurement of pylon height, distance between power lines and distance from power line to vegetation, etc. These tools can be very useful for indoor inspection of transmission line.

Except for manual distance measurement between transmission components and surrounding objects, some automatic and practical tools could be revealed based on these 3D models. Vegetation encroachment occupies the majority of workload in power line cursing and because of the elongated structure of transmission lines, automatic detection of vegetation encroachment is one of the most meaningful and useful function. Based on these reconstructed geometrical information, the problem of vegetation encroachment could be transferred as closest distance calculation between sample points of power line and terrain models, which could be achieved with absolute automation. Some other related applications can be exploited based on these 3D models.

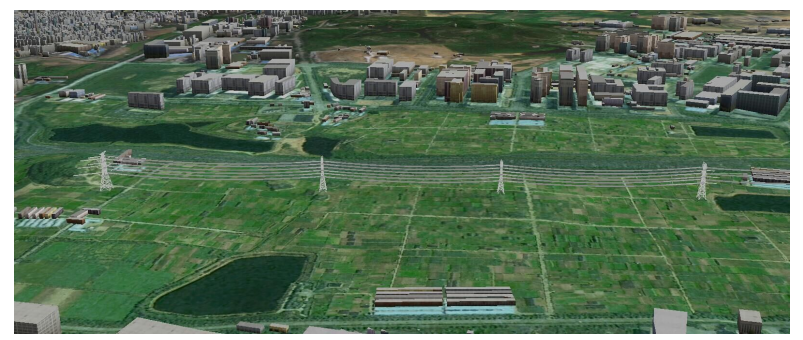

(a) Visualization of power pylons and lines

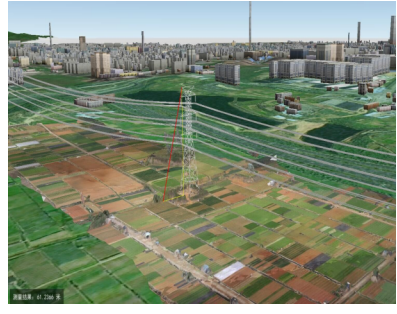

(b) Height measurement

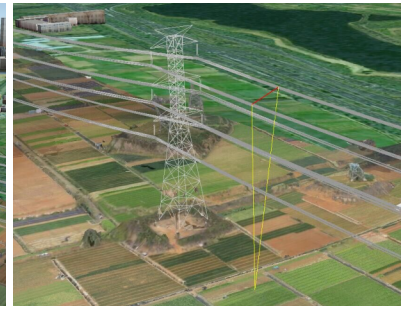

(c) Distance measurement
Figure 9. Applications based on reconstruction 3D models.

\section{CONCLUSIONS AND FUTURE STUDIES}

In this study, we have designed a dual-camera imaging system for data acquisition of transmission line and proposed a 3D model reconstruction solution for the purpose of transmission line inspection. Cooperating with the dual-strip flight mode, the oblique photogrammetric system can capture images of power pylons from four different directions, which provide enough information for 3D model reconstruction. From the comparison experiments between single-strip and dual-strip flight modes, we can see that dual-strip flight mode is recommended for inspection without GCPs because high geo-referencing accuracy could be achieved. With the dual-camera system and dual-strip flight mode, 3D model of corridor terrain as well as power pylons and lines have been successfully reconstructed.

Although only manual digitization of power pylons and lines is presented until now in this study, a more convenient and intelligent solution can be promoted with some extra functions developed in the future, such as automatic power line extraction with fewer user selected seed points and steel structure detection to assist power pylon reconstruction. Besides, because of the high resolution and overlap degree of oblique UAV images, acceleration for feature matching should be further improved to process larger datasets.

\section{ACKNOWLEDGEMENTS}

The authors would like to thank authors who have made their algorithms of SiftGPU and MicMac as free and open-source software packages, which is really helpful to this research.

\section{REFERENCES}

Ahmad, J., Malik, A. S., Xia, L. and Ashikin, N., 2013. Vegetation encroachment monitoring for transmission lines right-ofways: A survey. Electric Power Systems Research 95(1), pp. 339352.

Boccardo, P., Chiabrando, F., Dutto, F., Tonolo, F. G. and Lingua, A., 2015. Uav deployment exercise for mapping purposes: Evaluation of emergency response applications. Sensors 15(7), pp. $15717-15737$.

Gerke, M., 2009. Dense matching in high resolution oblique airborne images. In: U. Stilla, F. Rottensteiner and N. Paparoditis (eds), CMRT09: Object extraction for 3D city models, road databases and traffic monitoring : concepts, algorithms and evaluation, Paris, 3-4 September 2009. I ed by U. Stilla, F. Rottensteiner and N. Paparoditis. ISPRS, 2009. pp 77-82, International Society for Photogrammetry and Remote Sensing (ISPRS), pp. $77-82$.

Gerke, M. and Kerle, N., 2015. Automatic structural seismic damage assessment with airborne oblique pictometry imagery. Photogrammetric Engineering and Remote Sensing 77(9), pp. 885-898.

Gonçalves, J. A. and Henriques, R., 2015. Uav photogrammetry for topographic monitoring of coastal areas. ISPRS Journal of Photogrammetry and Remote Sensing 104, pp. 101-111.

Jiang, S., Jiang, W., Huang, W. and Yang, L., 2017. Uav-based oblique photogrammetry for outdoor data acquisition and offsite visual inspection of transmission line. Remote Sensing.

Katrasnik, J., Pernus, F. and Likar, B., 2009. A survey of mobile robots for distribution power line inspection. IEEE Transactions on Power Delivery 25(1), pp. 485-493.

Lin, Y., Jiang, M., Yao, Y., Zhang, L. and Lin, J., 2015. Use of uav oblique imaging for the detection of individual trees in residential environments. Urban Forestry and Urban Greening 14(2), pp. 404-412. 
Nex, F. and Remondino, F., 2014. Uav for 3d mapping applications: a review. Applied Geomatics 6(1), pp. 1-15.

Pierrot Deseilligny, M. and Clery, I., 2012. Apero, an open source bundle adjusment software for automatic calibration and orientation of set of images. ISPRS - International Archives of the Photogrammetry, Remote Sensing and Spatial Information Sciences XXXVIII-5/W16(5), pp. 269-276.

Rau, J. Y., Jhan, J. P. and Hsu, Y. C., 2014. Analysis of oblique aerial images for land cover and point cloud classification in an urban environment. IEEE Transactions on Geoscience and Remote Sensing 53(3), pp. 1304-1319.

Remondino, F. and Gerke, M., 2015. Oblique aerial imagery-a review. Proc. Week pp. 75-83.

Rupnik, E., Nex, F., Toschi, I. and Remondino, F., 2015. Aerial multi-camera systems: Accuracy and block triangulation issues. ISPRS Journal of Photogrammetry and Remote Sensing 101, pp. 233-246.

Sun, C., Jones, R., Talbot, H., Wu, X., Cheong, K., Beare, R., Buckley, M. and Berman, M., 2006. Measuring the distance of vegetation from powerlines using stereo vision. ISPRS Journal of Photogrammetry and Remote Sensing 60(4), pp. 269-283.

Vetrivel, A., Gerke, M., Kerle, N. and Vosselman, G., 2015. Identification of damage in buildings based on gaps in $3 \mathrm{~d}$ point clouds from very high resolution oblique airborne images. Isprs Journal of Photogrammetry and Remote Sensing 105, pp. 61-78.

Wu, C., 2007. Siftgpu: A gpu implementation of david lowe's scale invariant feature transform (sift). https://github.com/pitzer/SiftGPU (June 19 2017).

Yalcin, G. and Selcuk, O., 2015. 3d city modelling with oblique photogrammetry method. Procedia Technology 19, pp. 424-431.

Zhu, Q., Jiang, W. and Zhang, J., 2015. Feature line based building detection and reconstruction from oblique airborne imagery. International Archives of the Photogrammetry, Remote Sensing and Spatial Information Sciences 40(4), pp. 199.

\section{APPENDIX (OPTIONAL)}

Any additional supporting data may be appended, provided the paper does not exceed the limits given above. 